\title{
Multichannel Raman Gas Analyzer: The Data Acquisition and Control System. Measurement Improvement With Blue Laser Light
}

\author{
Panagiotis G. Papageorgas, Dimitris Maroulis, Harald Winter, Stavros A. Karkanis, Hansjörg Albrecht, and \\ Nikiforos G. Theofanous
}

\begin{abstract}
In this paper, the data acquisition and control system of a multichannel Raman effect-based gas analysis device is presented, together with the improvements achieved in measurement of gas concentration sensitivities as a result of the operation of the system with a new blue laser-light source. The multichannel Raman gas sensor (MRGS) is based on the linear Raman scattering effect and uses photo multiplier tubes (PMTs) in the photon-counting mode of operation. An embedded microcontroller-based data acquisition and control (MDAC) system collects, digitizes, processes, and stores in real time the data from six photon-counting modules and the accompanying sensors, along with an overall system control through appropriate actuators. Furthermore, the MDAC system supports the remote operation of the MRGS device from a host computer through a serial link for the modification of the operating parameters, the downloading of new software versions, and the uploading of the collected data for further analysis. The overall device is controlled by the MDAC system and can operate daily, either manually or in a completely automated mode. Many software and hardware features of the MDAC system described in this article can be adapted to other microcontroller-based acquisition and control systems. Recent advances in the development of solid-state laser sources have enabled the use of a new, state-of-the-art, blue laser for the excitation of the Raman effect. Using this blue laser source, improvements in the sensitivities in measurements of concentration for all tested gases $\left(\mathrm{SO}_{2}, \mathrm{CO}_{2}, \mathrm{CO}, \mathrm{NO}_{2}, \mathrm{C}_{6} \mathrm{H}_{6}\right.$, and $\mathrm{N}_{2}$ ) have been substantiated, compared with the green laser source previously used and reported in a related article.
\end{abstract}

Index Terms-Air-pollution, data acquisition, gas analysis, microcontroller, photon counting, Raman spectroscopy, real-time processing.

\section{INTRODUCTION}

A NUMBER of methods are currently utilized in commercial systems that are measuring the concentration of various gases. These methods can be categorized into those that are used for single gas measurement and those used for multiple gas measurement. The methods employed for single gas measurement are IR absorption, electrochemical, Raman, and thermo-conductivity. For multiple gas measurement, the methods employed are gas chromatography, IR absorption,

Manuscript received March 1, 2003; revised August 30, 2003. This work was supported in part by the European Commission.

P. G. Papageorgas, D. Maroulis, S. Karkanis, and N. G. Theofanous are with the Department of Informatics and Telecommunications, University of Athens, Athens, Greece.

$\mathrm{H}$. Winter and $\mathrm{H}$. Albrecht are with the Laser-und Medizin-Technologie GmbH, Berlin, Germany.

Digital Object Identifier 10.1109/TIM.2003.822012
FTIR (absorption), Raman, and a multimethod that utilizes the best method for each gas measured.

An efficient and advantageous method among the above-listed is the Raman method, which is used for simultaneous monitoring of a number of air pollutants in the multichannel Raman gas sensor (MRGS) device recently conceived and realized by the authors within the framework of a European Commission project. This pollution sensor, using a diode laser light source, is based on an exploitation of the Raman scattering effect [1]-[4] combined with appropriate optoelectronic data acquisition and signal handling techniques [5], [6].

The MRGS system utilizes the Raman scattering signal that is analogous to the gas concentration of interest, as the intensity of the exciting laser power is not large enough to cause resonance or nonlinear Raman effects. For linear Raman scattering, the intensity of the Raman scattering signal can be expressed as follows [7], [8]

$$
I_{R}=I_{0} \bullet N \bullet L \bullet E \bullet \Omega \bullet\left(\frac{d \sigma_{L}}{d \Omega}\right) \bullet\left(\frac{\nu_{0}-\nu}{\nu_{L}-\nu}\right)^{4} .
$$

In this equation, $\nu_{0}$ is the frequency of exciting laser light, $\nu_{L}$ is the reference frequency (here, $5145 \mathrm{~nm}$ ), $\nu$ is the corresponding Raman shift, $I_{R}$ is the intensity of the Raman scattered light, $\mathrm{I}_{0}$ is the intensity of the exciting Laser, $\mathrm{N}$ is the Molecule concentration of the measured gas (parts per $\mathrm{cm}^{-3}$ ), $\mathrm{L}$ is the scattering length, $\mathrm{E}$ is the detection efficiency, $\Omega$ is the solid angle [sr], and $\left(d \sigma_{L}\right) /(d \Omega)$ is the differential scattering cross section of the gas measured at the reference laser line $\left[\mathrm{cm}^{2} / \mathrm{sr}\right]$.

From the above equation, we can observe that a linear relationship exists between the Raman scattering signal and the relative cross section of the detected gas as well a linear relationship with the frequency of the exciting laser source $\nu_{0}$. The relative cross sections for the gases measured with the system developed are 0.93 for $\mathrm{CO}, 1.13$ for $\mathrm{CO}_{2}, 3.99$ for $\mathrm{SO}_{2}, 12.4$ for $\mathrm{C}_{6} \mathrm{H}_{6}$, and 13 for $\mathrm{NO}_{2}$ [9], [10]. It is, thus, expected that the sensitivities of different gases will vary according to their relative cross section. It is also expected that the use of a blue laser source $(457 \mathrm{~nm})$ will result to higher Raman scattering signal and thus improved sensitivities when compared with the sensitivities achieved with the green $(532 \mathrm{~nm})$ laser source since the frequency of the blue laser is higher according to (1). This was the main motivation for substituting the green laser source with 
a blue one as it is presented in this article in order to improve the gas measurement sensitivities.

A principal part of the MRGS setup is a specific multichannel photon counting (MPC) system that has been reported in [5] and [6]. In these references, the description was mainly focused on the electronics and signal-handling stages used for the realization of the photon-counting modules of the MPC system along with experimental results obtained using a green laser source at $532 \mathrm{~nm}$.

The other important part of the MRGS device is the system that performs the control of the entire sensor and acquires the corresponding gas concentration data combined with time-stamp and environmental conditions information. More precisely, these combined operations are realized on the basis of an embedded microcontroller that effects data acquisition, from the output of the MPC unit and preprocessing and storage of measurements and results along with the control of the entire MRGS device. In what follows, we shall call this system the microcontroller-based data acquisition and control (MDAC) system.

In this paper, the above MDAC system is described, as part of the entire MRGS setup, with its structure and hardware given together with the corresponding support software. In addition, the experimental operation and performance characteristics of the MDAC system are presented here, in the framework of an MRGS device improved by the use of a blue laser beam at $457 \mathrm{~nm}$ instead of the green one. This improvement, already come into sight from the work of [5], is now substantiated and documented with precise and extended results.

\section{OVERVIEW DEVICE DESCRIPTION}

The actual multichannel Raman gas sensor (MRGS), embodies the MDAC system under consideration as an important constituent and supporting part and has been implemented using most of units described in [5] and [6] but in a more integrated and improved form. The entire device mainly comprised the laser source, a multipass optical resonator arranged in a specific cuvette containing the gases under reference or test, six optical channels followed by corresponding photon-counting modules/units, appropriate data-acquisition and control units, and the indispensable sensors, and actuators along with the signal conditioning electronics. A simplified block diagram of the MRGS device (showing roughly the more important units of the MDAC system), is depicted in Fig. 1. For more details on the structure and operation of the multichannel photon counting system and the associated electronics, the reader should be addressed to [5] and [6]. In the following sections, a detailed description of the actual MDAC system is presented along with the supporting software used in the device.

It is to be pointed-out again that, by contrast to the previous version reported, where the laser source mainly used was a 532-nm green laser, in the present version, a 457-nm blue laser beam has been exclusively used for achieving improved results. As it is proved, the use of a blue laser light improves significantly the measurement sensitivity for all of the six gases of experimentation, which are $\mathrm{SO}_{2}, \mathrm{CO}_{2}, \mathrm{CO}, \mathrm{NO}_{2}, \mathrm{C}_{6} \mathrm{H}_{6}$, and $\mathrm{N}_{2}$. The later gas has been employed as a basis of reference

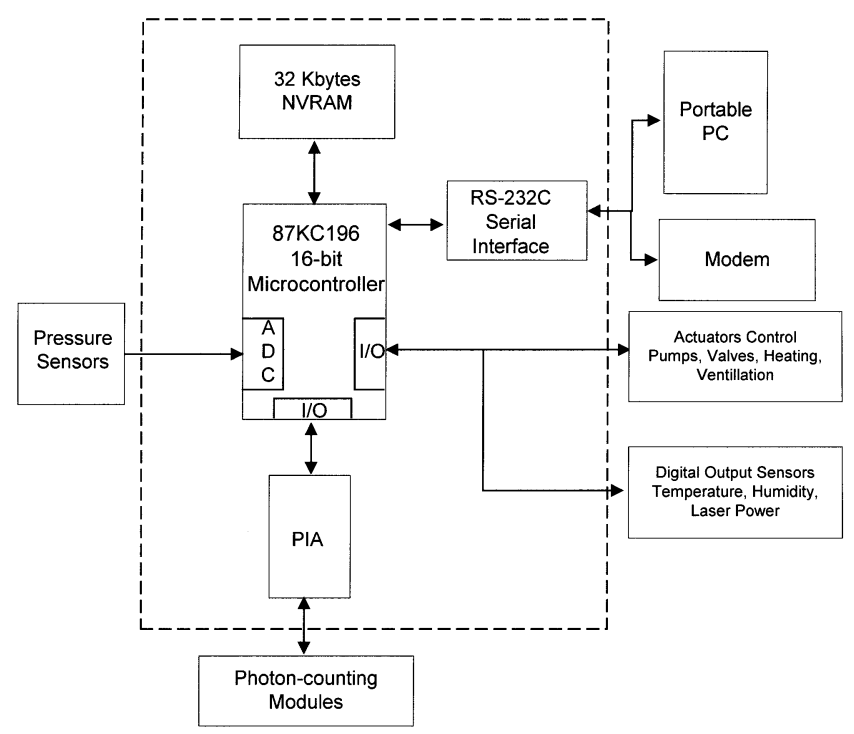

Fig. 1. Block diagram of the single board data acquisition and control system.

for the entire setup. In the practical device, separate PMTs and Raman selection optical filters have been used for the six channels of the apparatus. More specifically, three interference filters has been used per channel, viz., one high-pass filter for the suppression of elastically scattered light, via Rayleigh and Mie scattering, and two bandpass filters for the selection of the specific Raman wavelength and additional suppression of elastically scattered light. The foregoing filters (one composite filter for each gas component) are combined with f/1 optics for focusing the resulting light on the photocathodes of PMTs. In addition, independent signal amplification, discrimination from noise, and counting of pulses (photons) is performed on the output of each channel.

\section{DATA ACQUISITION AND CONTROL SYSTEM}

The MDAC system under consideration has been developed as a compact single board resident in the MRGS device. This system is based on an appropriate microcontroller and has been designed to provide and ensure increased versatility for the MRGS setup by assigning all of the main operating functions under software control. The user can operate and program the MRGS system with a portable PC, locally or remotely connected through a serial interface. It is also of note that an upgrading of the MDAC system is achieved by using nonvolatile memory for the storage of the operating software.

Microcontroller-based data acquisition systems are routinely reported in the literature and in various scientific fields [11]-[14], but usually their description is not focused on the indispensable details of the hardware and software developed. In the present section a description of the basic MDAC hardware structure and operation is made with a brief presentation of the microcontroller program memory options considered for application in the system.

The main part of the MDAC system is the Intel $87 \mathrm{KC} 196$ that belongs to the MCS96 family of microcontrollers. The MCS96's register-based architecture reduces accumulator bottlenecks and enables fast context switching that is important 
for real-time applications. This microcontroller integrates in one chip a 16-bit CPU core, 16 KB of OTP ROM, a number of timers and digital ports, a full-duplex asynchronous serial port, and an eight-channel/10-bit A/D converter. In the OTP ROM part of the microcontroller a tiny software kernel is stored that supports the downloading of new versions of software for instrument operation and modifications of system parameters in the nonvolatile RAM (NVRAM) memory, using the on-chip asynchronous serial communication via a host computer.

Contemporary trends for the nonvolatile storage of microcontroller programs are 1) the use of serial communication EEPROMS, 2) the use of flash-based microcontrollers or flash memories, and 3) the choice of a nonvolatile RAM realized either by a back-up battery or by an EEPROM. For the MDAC system of the present work, we have adopted the third solution, i.e., a nonvolatile RAM has been employed in which the RAM contents are preserved during the power-up and power-down cycles. This choice has been mainly made because of the simplicity of the supporting hardware and software applied for the reprogramming procedure compared with the alternative solutions referenced. For the NVRAM memory, the Dallas DS1386-32 chip has been selected, which integrates in one module a static RAM of $32 \mathrm{~KB}$, a real-time clock and a watchdog programmable timer, along with the battery needed for the operation of the clock and the retention of RAM data. The NVRAM memory is logically separated into two blocks: the first $4 \mathrm{~KB}$ are used for the application program while the remaining $28 \mathrm{~KB}$ are dedicated for the storage of the acquired data. Note that the MDAC software is stored in the NVRAM, along with the operating parameters and the results of measurements performed.

Following power-up, the microcontroller operation is initialized by the bootstrap program stored in the OTP ROM and the execution of the main program starts with the transfer of control to the specific code area of the NVRAM section. Subsequent downloads of software upgrades can be performed in the field using the RS-232 interface. This feature constitutes one of the more significant advantages of the MDAC system, because it permits the future expansion or modification of the system to be easily performed without any need for major hardware changes.

The description that follows refers to the schematic diagram of the MDAC system depicted in Fig. 2. The watchdog timer output (INTA) resets the whole system via the appropriate microcontroller input (RESET) in case of a software fault. For this operation a time delay is programmed (in steps of $10 \mathrm{~ms}$ ) after the expiration of which a reset of the microcontroller is performed. In the normal software operation, this watchdog timer is reprogrammed before the expiration of the predefined time interval. Also, the integrated real-time clock enables the programming of time intervals in steps of $2 \mathrm{~ms}$ with a dedicated counter. This counter is used for the determination of the counting interval of the photon-counting modules. For this purpose, the output pin of the counter (INTB) on the NVRAM chip is connected with the external interrupt input of the microcontroller (EXINT pin P2.2) and the control input (STOP) that supervises the counting operation of the photon-counting modules. With this arrangement, when the programmed time interval expires, an interrupt request is asserted to the microcontroller, in re- sponse to which the appropriate software routine is called for the acquisition of data from the photon-counting modules.

In the schematic diagram of Fig. 2, a number of glue-logic chips can be identified. A 74373 IC latch circuit is used for the demultiplexing of the address/data lines, which the specific microcontroller multiplexes in order to reduce the pin count. A 74138 decoder is employed for the decoding of memory addresses needed for referencing the various I/O peripherals of the MRGS system. As the address space of the 8K7C196 microcontroller is $64 \mathrm{~KB}$ and the NVRAM has a capacity of $32 \mathrm{~KB}$, we have adopted a simple technique that reserves large address spaces for address mapping of specific peripherals. For this purpose, the lower $32 \mathrm{~KB}$ of address space are allocated to the NVRAM and the upper $32 \mathrm{~KB}$ are allocated to the I/O peripherals. The 82C55 IC is the well-known peripheral interface adapter (PIA) that offers three bidirectional digital ports, each 8-bits wide. The PIA chip is used for increasing the number of digital I/O ports of the microcontroller in order to access the six photon-counting modules. These modules are accessed using the PIA chip of the MDAC system, which provide the necessary addresses under the control of the acquisition software. These specific addresses are sequentially generated from the six digital outputs of the PIA unit and the data corresponding to the counters of each specific module are first latched with the eight digital inputs of the same PIA and then are transferred to the microcontroller for processing and storage.

\section{SYSTEM SOFTWARE}

The entire MRGS device is supported by specific software, that has been developed mainly in assembly language for the microcontroller used and partially in $\mathrm{C}$ language. This software integrates the data acquisition and data processing operations, as well as the communication and control functionality of the overall system, together with the necessary user interface. The total software, as finally developed, has been divided into two separate "logical" parts. The first part is the software of the microcontroller-based data acquisition and control system (MDAC executed software) while the second one is the software corresponding to the host computer for supporting the remote control operation of the entire system (host PC-executed software).

\section{A. MDAC-Executed Software}

This software has been exclusively developed in assembly language in order to achieve high-speed execution of the involved functions and to offer the real-time performance to our system. This software supports the control of the MDAC system along with the concurrent execution of the following three tasks performing:

- acquisition of the photon-counting data;

- preprocessing and storage of the acquired data;

- remote control of the system using the asynchronous communication interface.

The real-time execution of these tasks is provided by the kernel software stored in the OTP memory of the microcontroller unit, by using its interrupt mechanism and realizing a simple multitasking system capable of servicing the critical timing needs of the system with a worst-case interrupt latency 


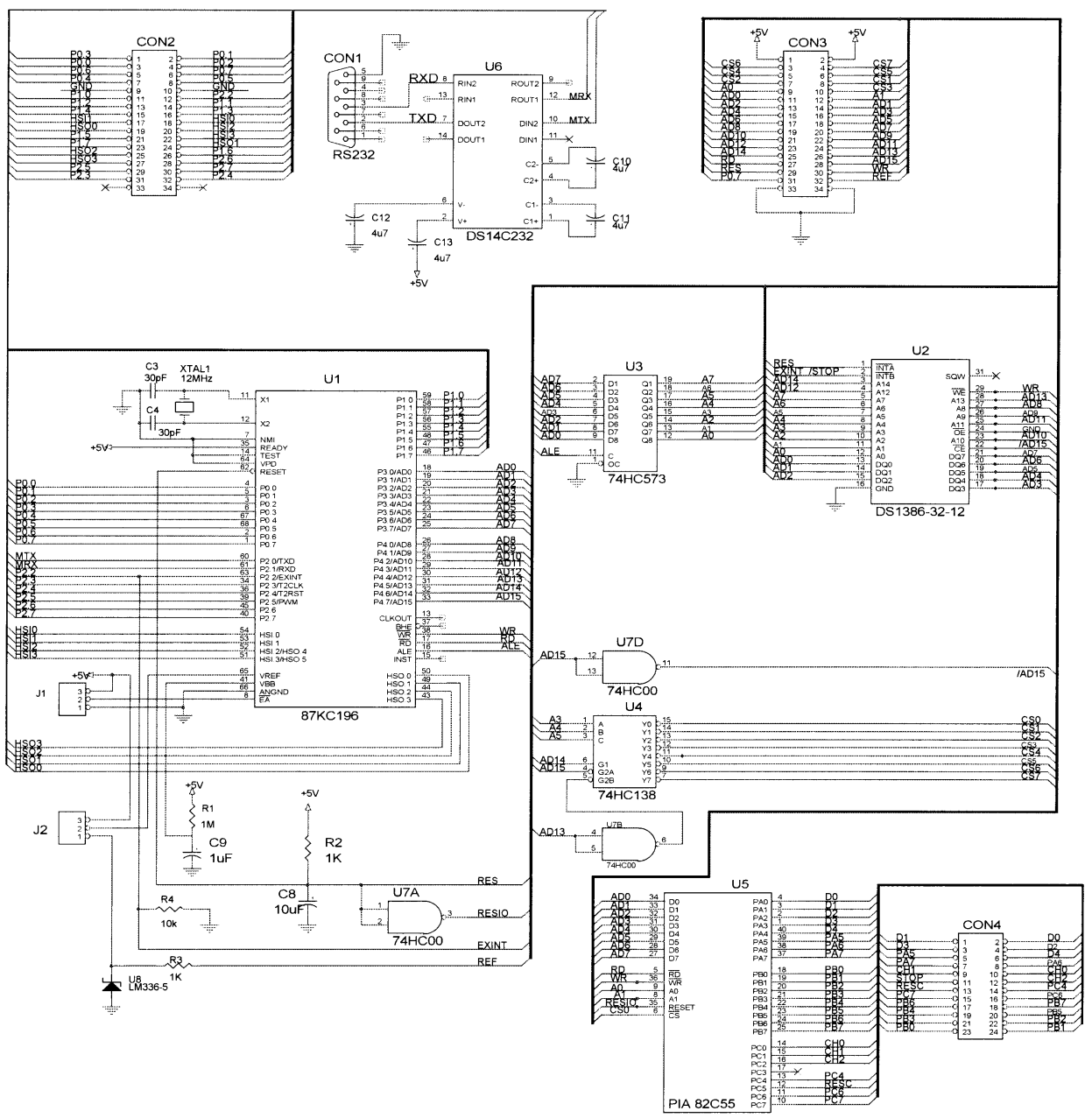

Fig. 2. Schematic diagram of the single board MDACS.

of $2 \mu \mathrm{s}$. The above three tasks are serviced in a preemptive event-driven mode.

The operation of the microcontroller software can be well described by means of the complete state diagram depicted in Fig. 3. On the other hand, the structure of this software is divided into the following two major sections/modules ensuring flexibility according to the user needs and any future instrument modifications or extensions.

1) The first section is a small kernel module that is stored in the one-time programmable memory (OTP PROM) of the microcontroller. This kernel supports the basic operations of the MDAC system, such as the initialization routine, the remote control of the device and the downloading routine. With the initialization routine, that is executed during the power-up procedure, the transfer of all interrupt vectors from the internal OTP ROM addresses to external NVRAM addresses is performed, together with the reset operation of the photon-counting modules and the actuators used. Moreover, the downloading routine supports modifications of the MDAC system's operating software via its asynchronous serial interface, as well as the multitasking operation and the remote control of the system using a command parser, which has been also developed and integrated into this kernel.
2) The second section is the main software module of the microcontroller unit. This module is stored in the NVRAM and supports the data collection, the reset operation, and the preprocessing and storage of the photon-counting measurements. Also, this module asserts the transfer of the corresponding data to the host system. In the event of detection of activity in the asynchronous serial interface, the control is transferred to the command parser program located in the OTP memory area that executes the valid remote control commands.

The software code downloaded and the collected data uploaded are packed using the typical INTEL HEX format. This format has been chosen for its structural simplicity, for the error identification mechanism supported and, more significantly, for its wide acceptance as the output format by most software vendors developing assemblers and higher-level language compilers for the specific microcontroller.

The command parser developed, supports the remote operation of the system and is partly integrated into the kernel module of the microcontroller operating software while the commands supported are realized in the NVRAM memory, for increased versatility. This parser is activated with the presence of valid characters at the asynchronous serial-input port of the system. As soon as a valid command is recognized, it is immediately 


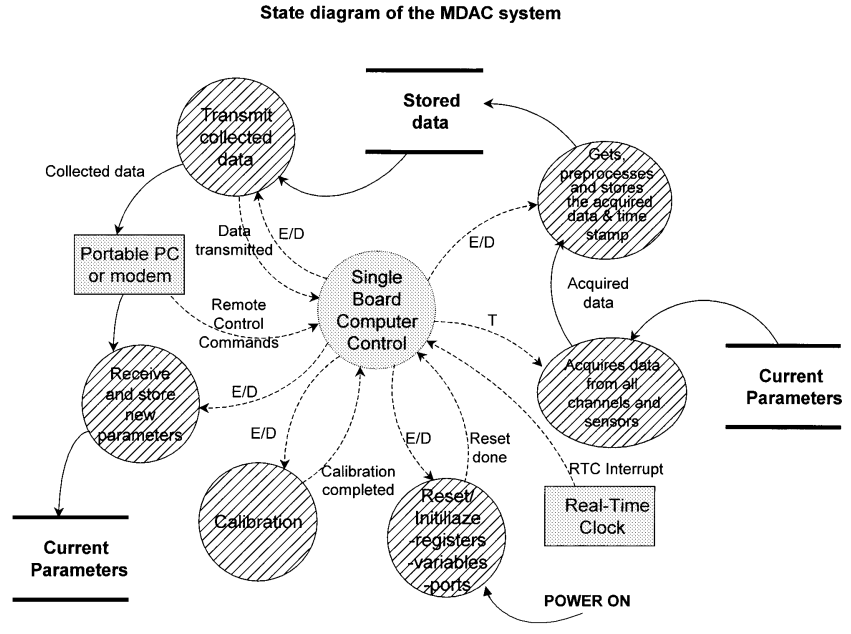

Fig. 3. State diagram for the microcontroller software.

serviced with the transfer of control to the appropriate subroutine and the execution of the corresponding operations. With this software module, the following basic remote commands have been developed for the specific application:

- downloading of new software versions;

- real-time date and time settings;

- configuration of operating parameters;

- realization of calibration procedure;

- reset operation;

- uploading of the acquired data.

During the system's power-up, the execution of the initialization subroutine is first performed. After its completion, the control is transferred to the main software module where the preprocessing and storage of the acquired photon-counting data is effected. An interrupt trigger provided by the real-time clock chip starts a new acquisition cycle. This interrupt triggers the acquisition operation of the photon-counting modules by passing the control to the appropriate software routine. In addition, the detection of activity in the asynchronous serial input has as result the transfer of control to the command parser routine, where the validity of the command is first verified and then the associated routine is called for execution.

\section{B. Host PC-Executed Software}

The second "logical" part of the developed software that is executed at the host PC-type computer, supports further "off line" data processing and provides a full-duplex communication with the MRGS setup. The main operations that this software realizes are: a) the mass storage of the acquired data, b) graphical user interface for the remote operation of the entire MRGS instrument, c) further data processing, and d) the graphical presentation of the acquired data.

The implemented host-PC software supporting the above functions can be further divided into two main sections. The first of them performs modification of the operating parameters, if needed, or the uploading of new software versions of the MRGS device and the second one effects the transfer of the acquired measurements to the host computer, for mass storage, further analysis and final presentation. Also, a graphical user interface has been developed that integrates the above features using the Labwindows-CVI software development platform from National Instruments. All of the known conventions for the Microsoft Windows operating system, as regards the file open, name and storage operations, along with the menus used for specifying the necessary operating parameters, have been considered during the above software development.

In the state diagram shown in Fig. 3, the execution steps of the software developed are illustrated in details. At first, the serial communication is established between the PC and the MDAC system. The next possible step is the calibration of the indispensable sensors that can be done using the appropriate settings, stored in a specific parameter file in conjunction with those specified during the last operation of the system. The settings for the calibration of the device along with the new operating parameters are transferred to the MDAC system using the Intel Hex format, which provokes a reset operation of the MRGS setup.

The PC host computer receives the acquired data that are transmitted via the asynchronous output of the MDAC system. These data represent the results of the photon-counting measurements accompanied by the associated data extracted from the sensors and followed by a time stamp that defines the time interval in which each measurement has been performed. The data in question, which are stored in the hard disk of the host computer, are plotted on the six strip-chart recorder implemented in the main panel of the application software. In this main window panel, specific virtual buttons have been integrated allowing the user to select various operations such as downloading and saving in a file the data acquired for a specific time period, uploading new versions of the operating software, modifying the operating parameters and activating the reset/calibration operations. Also, additional similar buttons have been installed for commanding various desired activations and deactivations of the MRGS device actuators. Each of these buttons has been associated with a specific command the code of which is packed in the Intel Hex format and then is uploaded through the serial interface to the MDAC system, which in turn acknowledges to the host PC the reception and execution of the command.

\section{Sensitivity of Concentration Measurements USING THE BLUE LASER BEAM}

The MDAC system described was extensively tested in the laboratory, in the framework of a MRGS device, using gas samples (test gases) with known concentrations. To improve sensitivity, in the present version the green-light laser source, employed in the work of [5] and [6], has been substituted with a blue laser, that has been provided to us as a commercial prototype from the manufacturing company LASER POWER Ltd. with the specifications given in Table I.

The use of the above blue laser in the MRGS device, to ensure increased sensitivities for measurements of the gases of interest, implied a number of modifications, e.g., the gasspecific interference filters and the spherical mirrors forming the multipass resonator were changed to be adapted to the new wavelength. Also, a most suitable integration time for this new setup was determined and found to be 30 s. Finally, 
TABLE I

SPECIFICATIONS OF BLUE LASER

\begin{tabular}{l|l|l}
\hline & $\begin{array}{l}\text { Serial Number } \\
\mathbf{1 0 7}\end{array}$ & Comments \\
\hline Wavelength $[\mathrm{nm}]$ & $457 \pm 0.5$ & blue \\
\hline Output Power $[\mathrm{mW}]$ & 100 & nominal $-\mathrm{CW}$ \\
\hline Beam Diameter $(1 / \mathrm{e} 2)[\mathrm{mm}]$ & 0.25 & beam waist \\
\hline Beam Divergence $(\mathrm{Half}$ angle $[\mathrm{mrad}]$ & $<10$ & \\
\hline Polarization Ratio & $>100: 1$ & horizontal \\
\hline Pointing Stability $[\mathrm{mrad} / \mathrm{hr}]$ & $<0.2$ & after 60 sec warm-up \\
\hline Amplitude Stability $[\%]$ & $< \pm 3$ & after 60 sec warm-up \\
\hline Mode & Multi-Mode & \\
\hline Temperature Range $\left[{ }^{\circ} \mathrm{C}\right]$ & $10-40$ & Operating \\
\hline
\end{tabular}

each measurement sequence for determining the sensitivity was composed of five steps with each step consisting of 50 time windows, each one possessed a counting time of $30 \mathrm{~s}$. The above steps correspond to the following rates: dark count rates, count rates for stray light (non-Raman active gas), count rates for test gas mixture, count rates for stray light, and dark count rates again. In the present set of measurements, an Argon gas was used as a base-line (background) test gas because, as being a monatomic gas, it does not produce Raman scattering and therefore provides the reference level for the measurements performed.

It is also important to note that, to make comparisons on a commercial basis of reference offering highest sensitivity and repeatability, in the actual setup, the MDAC system has been combined with the commercially available photon-counting system SR400 from Stanford Co.

\section{A. Sensitivity for $\mathrm{SO}_{2}$ Channel}

Fig. 4 shows the results of measurements effected in order to determine the $\mathrm{SO}_{2}$-channel sensitivity by using a test gas with known $\mathrm{SO}_{2}$ concentration. The left and the right axes shows the numbers of counts for nitrogen and sulfur dioxide gas, respectively. In both channels, the stray light levels, which are represented by the values obtained for the non-Raman active gas (Argon), were ascertained to be about the same. The stray light level has been found to be about two orders of magnitude higher than the dark-count levels. The dark count rate for the $\mathrm{SO}_{2}$ channel has been determined to be equal to $3.0 \pm 0.4 \mathrm{cps}$ and for the $\mathrm{N}_{2}$ channel $6.7 \pm 0.7 \mathrm{cps}$. Of course, the existence of a low dark count rate leads to increased sensitivity.

The sensitivity for the $\mathrm{N}_{2}$ channel was measured at approximately $1.0 \%$, whereas the sensitivity for the $\mathrm{SO}_{2}$ channel was measured at $177 \mathrm{ppb}$ (parts per billion). Two evaluations were made, the first one using the non-Raman active signals obtained before the test gas was introduced and the second one using the same signals but after the introduction of the test gas. Both sensitivities were found to be in the same range of values. The differences observed among them are explained by the time needed for $\mathrm{N}_{2}$ of the test gas to flush out of the cuvette gas cell. Note that the normalization as to the $\mathrm{N}_{2}$ channel was done under the assumption that the signal in the $\mathrm{N}_{2}$ channel is linearly proportional to the laser power. This normalization was effected by dividing each 30-s measurement of the $\mathrm{SO}_{2}$ channel with the corresponding 30-s measurement obtained for the $\mathrm{N}_{2}$-channel. In this way, errors due to fluctuations in the laser power are adequately minimized. As deduced from the obtained results,

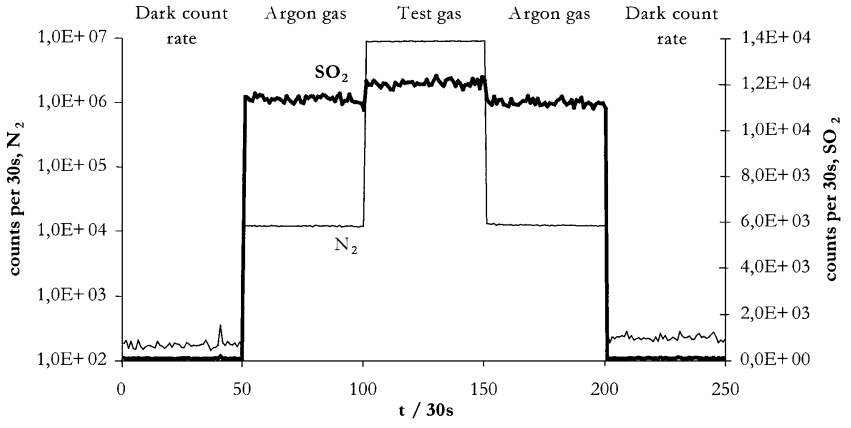

Fig. 4. $\mathrm{SO}_{2}$ measurements.

the sensitivity achieved for the $\mathrm{SO}_{2}$ channel with the present blue-laser setup is approximately $200 \mathrm{ppb}$ enhanced by a factor of nearly 400 compared to the case of the green laser excitation, with the sensitivity measured to be equal to approximately $75 \mathrm{ppm}$.

\section{B. Sensitivity for $\mathrm{CO}_{2}$ Channel}

Fig. 5 illustrates the results of measurements effected for determining the $\mathrm{CO}_{2}$-channel sensitivity by using a test gas with known $\mathrm{CO}_{2}$ concentration. The left and the right axes show the numbers of counts for nitrogen and carbon dioxide gas, respectively. Normalization was done by forming the ratio of the values obtained from the two channels (i.e., $\mathrm{CO}_{2}$ divided by $\mathrm{N}_{2}$ ). It can be noted that, again, quite some time is needed before the $\mathrm{N}_{2}$ of the test gas is flushed out of the cell. Therefore, in this series of measurements, the number of measurement windows was increased from 50 to 100 . The levels of values of both channels for the non-Raman active gas were found to be about the same. The stray light level has been found to be about 2 to 3 orders of magnitude higher than the dark count levels. The dark count rate for the $\mathrm{CO}_{2}$ channel was measured equal to $0.36 \pm 0.23 \mathrm{cps}$ and for the $\mathrm{N}_{2}$ channel $6.5 \pm 0.5 \mathrm{cps}$. The sensitivity of $1.2 \%$ found for the $\mathrm{N}_{2}$ channel is within reasonable limits and, with a sensitivity of $161 \mathrm{ppb}$ for the $\mathrm{CO}_{2}$ channel, the ppb region was reached again. Compared to previous measurements with green-laser excitation (where the sensitivity measured to be equal to approximately $35 \mathrm{ppm}$ ), the $\mathrm{CO}_{2}$ sensitivity is enhanced by about a factor of 200 .

\section{Sensitivity for $\mathrm{C}_{6} \mathrm{H}_{6}$ Channel}

Fig. 6 shows the results of the measurement sequence performed for determining the sensitivity for $\mathrm{C}_{6} \mathrm{H}_{6}$ channel by using a test gas with known $\mathrm{C}_{6} \mathrm{H}_{6}$ concentration.

This time, the levels of this channel for the non-Raman active gas (characterizing the stray light level) and the dark-count rate differ by a factor of almost 6 . This is due to the fact that the wavelength of the Raman line monitored for $\mathrm{C}_{6} \mathrm{H}_{6}$ is much closer to the exciting wavelength than any other Raman line of the other gases; thus the $\mathrm{C}_{6} \mathrm{H}_{6}$ channel proves to be more sensitive to the stray light. The stray light level in this case could be reduced by using a different Raman line of $\mathrm{C}_{6} \mathrm{H}_{6}$, notably the line of $3070 \mathrm{~cm}^{-1}$ (relative differential scattering cross section 15.4) instead of $992 \mathrm{~cm}^{-1}$ (relative differential scattering cross section 12.4). (The decrease in sensitivity of the Photomultiplier 


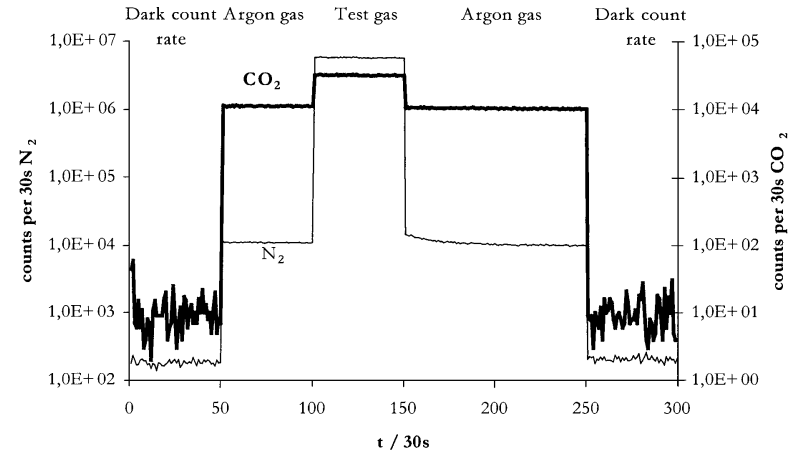

Fig. 5. $\mathrm{CO}_{2}$ measurements.

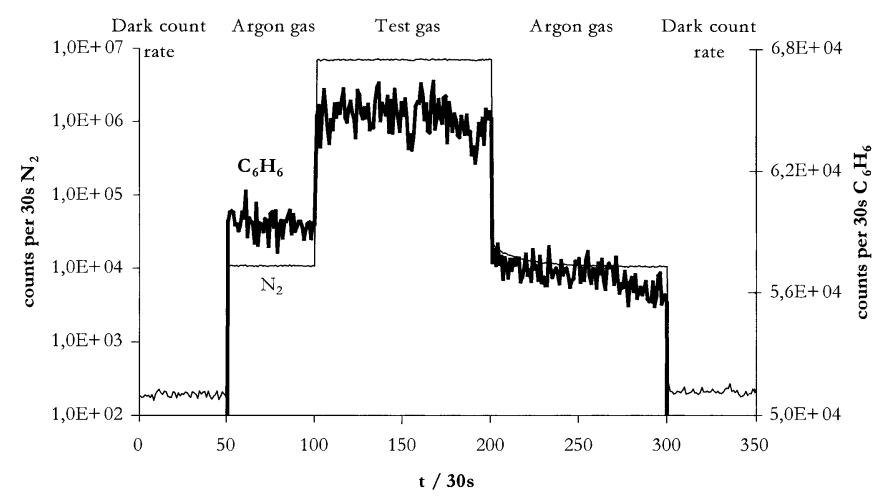

Fig. 6. $\mathrm{C}_{6} \mathrm{H}_{6}$ measurements.

Tubes as a result of higher wavelengths is partly compensated by the larger relative differential scattering cross section). The dark-count rate for $\mathrm{C}_{6} \mathrm{H}_{6}$ channel has been found to be $2.7 \pm$ $0.5 \mathrm{cps}$ and for $\mathrm{N}_{2}$ channel $6.7 \pm 0.5 \mathrm{cps}$, respectively. The sensitivities of $1.3 \%$ measured for the $\mathrm{N}_{2}$ channel and $1.2 \mathrm{ppm}$ for the $\mathrm{C}_{6} \mathrm{H}_{6}$ channel are both estimated as reasonable levels. Compared to previous measurements with green-laser excitation (where the sensitivity was measured equal to approximately $35 \mathrm{ppm}$ ), the sensitivity for $\mathrm{C}_{6} \mathrm{H}_{6}$ with blue-laser light is enhanced by a factor of about 28 .

\section{Sensitivity for $\mathrm{NO}_{2}$ Channel}

Fig. 7 shows the results of the measurement sequence performed for determining the $\mathrm{NO}_{2}$ sensitivity using test gas with known $\mathrm{NO}_{2}$ concentration. The dark-count rate for the $\mathrm{NO}_{2}$ channel has been found equal to $17.3 \pm 0.5 \mathrm{cps}$ and for the $\mathrm{N}_{2}$ channel $6.4 \pm 0.2 \mathrm{cps}$. With sensitivities of $0.7 \%$ measured for the $\mathrm{N}_{2}$ channel and $0.2 \mathrm{ppm}$ for the $\mathrm{NO}_{2}$ channel, these values appear both reasonable.

For $\mathrm{NO}_{2}$ no increase in sensitivity has been observed with the normalization as to $\mathrm{N}_{2}$, which means that the $\mathrm{NO}_{2}$ channel has a different behavior than the other channels. This fact can be explained again with the existence of a strong fluorescence in $\mathrm{NO}_{2}$; this fluorescence was observed even in the case of excitation with the green laser light (strong crosstalk). Compared to previous measurements with green-laser excitation (where the sensitivity measured to be approximately $0.5 \mathrm{ppm}$ ) the present sensitivity for $\mathrm{NO}_{2}$ has been found enhanced by a factor of about 2.

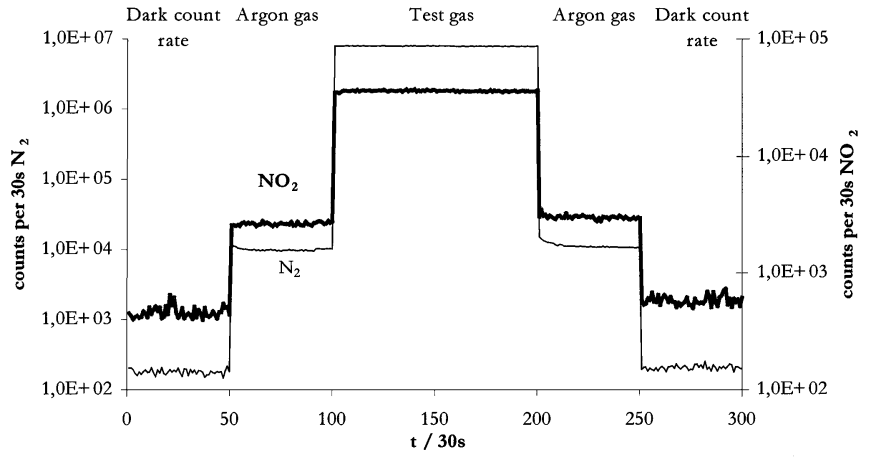

Fig. 7. $\mathrm{NO}_{2}$ measurements.

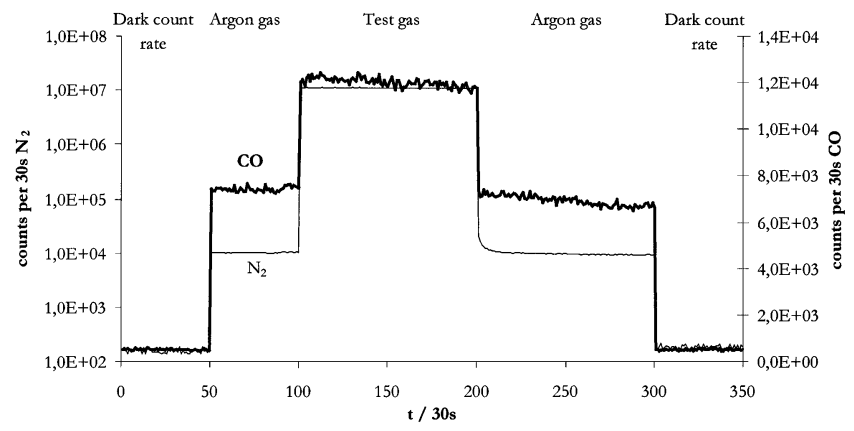

Fig. 8. $\mathrm{CO}$ measurements.

\section{E. Sensitivity for CO Channel}

Fig. 8 shows the results of the measurement sequence performed for determining the $\mathrm{CO}$ sensitivity by using a test gas with known $\mathrm{CO}$ concentration. It can be noted again that it takes quite some time before the $\mathrm{N}_{2}$ of the test gas is flushed completely out of the cell. Therefore, the amount of measurement points was extended again from 50 to 100 . On the other hand, it has been found that, now, the levels of values of both channels for the non-Raman active gas (characterizing the stray light level) are not the same. The level of value for the $\mathrm{CO}$ channel was lower (by a factor of about 0.8 ). This gives reason to believe that the CO-channel detector is less sensitive because of the higher dark-count rates. The dark-count rate determined for the $\mathrm{CO}$ channel was $17.1 \pm 0.8 \mathrm{cps}$ and for the $\mathrm{N}_{2}$ channel $5.7 \pm 0.5$ cps. The sensitivities measured were $0.7 \%$ for the $\mathrm{N}_{2}$ channel and $0.5 \mathrm{ppm}$ for the $\mathrm{CO}$ channel. Compared to previous measurements made with green-laser excitation (where the sensitivity measured to be equal to approximately $890 \mathrm{ppm}$ ), the sensitivity has been found enhanced by a factor of about 445 .

\section{Consolidated Remarks on the System Sensitivities WITH BLUE-LASER EXCITATION}

From the obtained results, one can conclude that, by employing a blue micro-laser in the system, its performance is upgraded and the sensitivities of most channels are drastically improved. For better comparison, a summary of the results and improvements achieved by the use of the blue laser is given in Table II.

As seen from the table, for the $\mathrm{SO}_{2}$ and the $\mathrm{CO}_{2}$ channels, it was possible to achieve sensitivities lying in the ppb range (about $200 \mathrm{ppb}$ ), which are highly improved compared to the 
TABLE II

SUMMARY OF RESULTS

\begin{tabular}{l|c|c|c|c}
\hline GaS & $\begin{array}{c}\text { Sensitivity } \\
\text { (green-laser) }\end{array}$ & $\begin{array}{c}\text { Sensitivity } \\
\text { (blue-laser) }\end{array}$ & $\begin{array}{c}\text { Sensitivity } \\
\text { Increase }\end{array}$ & Comment \\
\hline $\mathbf{N}_{\mathbf{2}}$ & $1.1 \%-25 \%$ & $\approx 0.5 \%$ & $2-50$ & O.K. \\
\hline $\mathbf{S O}_{2}$ & $\approx 75 \mathrm{ppm}$ & $\approx 200 \mathrm{ppb}$ & $3.8 \times 10^{2}$ & O.K. \\
\hline $\mathbf{C O}_{2}$ & $\approx 35 \mathrm{ppm}$ & $\approx 200 \mathrm{ppb}$ & $1.8 \times 10^{2}$ & O.K. \\
\hline $\mathbf{N O}_{2}$ & $\approx 0.5 \mathrm{ppm}$ & $\approx 0.2 \mathrm{ppm}$ & 2 & Strong cross talk \\
\hline $\mathbf{C}_{\mathbf{6}} \mathbf{H}_{\mathbf{6}}$ & $\approx 35 \mathrm{ppm}$ & $\approx 1.2 \mathrm{ppm}$ & $2.9 \times 10$ & O.K. \\
\hline $\mathbf{C O}$ & $890 \mathrm{ppm}$ & $\approx 2 \mathrm{ppm}$ & $4.5 \times 10^{2}$ & O.K. \\
\hline
\end{tabular}

case of green-laser excitation, where the sensitivities were in the range of $50 \mathrm{ppm}$. Also, the sensitivity for the $\mathrm{CO}$ channel has reached the lower ppm range (of about $2 \mathrm{ppm}$ ), which is highly lower than that of the green-laser case, in which a sensitivity of about $890 \mathrm{ppm}$ has been measured. As to the sensitivity of the $\mathrm{C}_{6} \mathrm{H}_{6}$ channel, it has been found to be also in the lower ppm range $(\sim 1.2 \mathrm{ppm})$, which is nearly 30 times better than the sensitivity of $35 \mathrm{ppm}$ measured in the case of the green laser excitation. Lastly, the sensitivity for the $\mathrm{NO}_{2}$ channel remained nearly the same with that achieved in the green-laser case (about $1 \mathrm{ppm}$ ). This behavior and the fact that a strong crosstalk was present in all other channels during previous measurements, made with green-laser excitation has been attributed to the existence of a wide-band fluorescence.

In the present case, crosstalk problems between the channels have been investigated qualitatively and the following results have been obtained: The presence of nitrogen (up to more than $80 \%$ ) or oxygen (about $20 \%$ ) did not cause problems of this kind in the determination of sensitivities for all of the measurement channels. The only gas causing strong crosstalk was nitrogen dioxide and this fact was not ascribed to Raman scattering but to wide-band fluorescence. During all experiments, the nitrogen channel showed a very stable behavior. The large improvements observed in the sensitivities for some gases, as a result of the change of the excitation from green to blue laser light, can be attributed to the following reasons:

- increase in Raman effect intensity (from $532 \mathrm{~nm}$ to blue $457 \mathrm{~nm}$ ), which yielded an improvement factor of about 2;

- increase of quantum efficiency of photo-detectors, which yielded an improvement factor of up to 5;

- increase in the optical output power of laser, which yielded an improvement factor of about 2;

- increase in the stability of laser output power, which lead to the remaining enhancement of up to two orders of magnitude.

To check the validity of the above reasons for the increased sensitivities appearing in the blue-laser case and to be ensured that the increased sensitivities are not due to parasitic effects (fluorescence, stray light production, etc.), the count rates for the non-Raman active gas were compared. The differences in count rates so observed were such that the stray light and fluorescence contributions should be considered as negligible.

\section{SUMMARY AND CONCLUSION}

A low-cost MDAC system has been developed and presented as the control and processing part of the MRGS device used for air-pollution measurements. The described MDAC system, with the associated software, constitutes an efficient realization of the computer module needed. The MDAC system acquires, digitizes, calibrates, processes and stores the data provided by the multichannel photon-counting system of the MRGS device. The part of the developed software that is executed in the host PC (which is connected locally or remotely with the MRGS device through its asynchronous interface) permits an efficient graphical presentation of the acquired results of measurements together with off-line data processing and mass storage for further analysis. The specific realization of the MDAC system possesses flexibility for software modifications, most of which can be made using the serial interface with the host PC and the storage of new versions of operating software in the NVRAM memory of the system.

As well as the MDAC system upgrading action on the performance and the versatility of the entire MRGS device, additional improvement has been found to be achieved by using a blue laser light (at $457 \mathrm{~nm}$ ) instead of the green one (at $532 \mathrm{~nm}$ ). A modular hardware and software architecture was followed, along with the use of industrial standard hardware and software platforms, whenever possible. This allowed an easy adaptation of our data-acquisition and control system to the modifications of the MRGS device needed for this change in wavelength.

The overall MRGS device performance, especially when improved with the blue excitation laser source, has shown that the present prototype can be evolved, with minor modifications, to a successful commercial system. By comparing the measured performance of the MRGS device (as depicted in Table II) with the officially allowable limits for the gases under consideration, in the ambient air, it has been concluded that the allowed upper-limit values can be attained. For carbon monoxide and benzene the corresponding lower-limit values have been also reached. The next step would be to further improve the sensitivities, by one or two orders of magnitude, in order to reach the 1/10 lower-limit values for all gases, providing a good resolution with such a system. Besides increasing the laser power, such an improvement is possible by redesigning electronics, the multipass cell, etc, in view of the following considerations.

- Reduction of stray light may lead to a performance improvement up to a factor of 100 .

- Increase of multipass path may yield an improvement factor of 2-4.

- Reduction of electronic noise may yield a sensitivity improvement by a factor of 2 .

- An increase in laser power can be achieved by using a fiber coupling between the laser head and the multipass cell, thus resulting in a performance increase of one order of magnitude.

- Additional improvement can be performed by reducing the dark count rate of the photomultipliers using appropriate cooling techniques.

With the above modifications in the MRGS device, the sensitivity for all gases could reach the lower ppb region, which implies that the entire system can compete with the existing commercial monitoring setups. The cost of such a multichannel gas analysis system is expected to be competitively low and, 
with its compact design, can constitute an attractive solution compared with the overall cost of the existing gas analysis stations. Note that, typically, the latter ones comprise a number of commercially available units (each of which measures the concentration of only one gas) and present increased complexity due to the necessary calibration and data collection procedures.

\section{REFERENCES}

[1] A. Weber, P. S. Porto, L. E. Cheesman, and J. J. Barrett, "High-resolution Raman spectroscopy of gases with cw-laser excitation," J. Opt. Soc. Amer., vol. 57, pp. 19-28, 1967.

[2] H. Albrecht, G. Müller, and M. Schaldach, "Application of laser Raman spectroscopy to medical diagnosis II," in Proc. 6th Int. Conf. Raman Spectroscopy, vol. 2, Bangalore, India, Sept. 4-9, 1978, pp. 526-527.

[3] P. C. Kumar and J. A. Wehrmeyer, "Stack gas pollutant detection using laser Raman spectroscopy," Appl. Spectrosc., vol. 51, no. 6, pp. 849-855, 1997.

[4] T. Doerk, J. Ehlbeck, H. Jedamzik, J. Hoschele, and J. Steinwandel, “Application of coherent anti-stokes Raman scattering (CARS) technique to the detection of NO," Appl. Spectrosc., vol. 51, no. 9, pp. 1360-1368, 1997.

[5] P. G. Papageorgas, H. Winter, H. Albrecht, D. Maroulis, and N. Theofanous, "A multichannel photon-counting system for gas analysis with Raman-scattering technique," IEEE Trans. Instrum. Meas., vol. 48, pp. 1166-1177, Dec. 1999.

[6] P. G. Papageorgas, D. Maroulis, S. Karkanis, N. Theofanous, H. Winter, and $\mathrm{H}$. Albrecht, "A specific multichannel photon-counting unit for airpollution measurement," in Proc. 6th IEEE Int. Conf. Electronics, Circuits and Systems, Pafos, Cyprus, Sept. 5-8, 1999.

[7] H. Albrecht, "Untersuchungen zur Anwendung der Raman-Spektroskopie in der Medizin," Ph.D. dissertation, 1976.

[8] G. Müller and E. Weimer, "Multipass Systeme für die Raman Spektroskopie," Optik, vol. 56, pp. 1-19, 1980.

[9] H. Winter, "Multichannel Raman Gas Analysis for Quantitative Monitoring of Environmental Air Pollution," Ph.D. dissertation, Free University of Berlin, Berlin, Germany, 1999.

[10] H. W. Schrötter and H. W. Klöckner, "Raman scattering cross sections in gases and liquids," in Topics in Current Physics: Raman Spectroscopy of Gases and Liquids, A. Weber, Ed. Berlin, Germany: Springer, 1979, pp. 123-166.

[11] E. Pattey, W. Royds, R. Desjardins, D. Buckley, and P. Rochette, “Application software for a data acquisition and control system for measuring atmospheric trace gas and energy fluxes," Comput. Electron. Agriculture 15, vol. 15, pp. 303-321, 1996.

[12] R. Mukaro and X. F. Carelse, "A microcontroller-based data acquisition system for solar radiation and environmental montitoring," IEEE Trans. Instrum. Meas., vol. 48, pp. 1232-1238, Dec. 1999.

[13] L. Gori, R. Tommasini, G. Cautero, D. Giuressi, M. Barnaba, A. Accardo, S. Carrato, and G. Paolucci, "An embedded control and acquisition system for multichannel detectors," Nucl. Instrum. Met. Phys. Res., vol. A 431, pp. 338-346, 1999

[14] D. Maroulis, G. Dumas, J. L. Bougeret, C. Caroubalos, and M. Poquerusse, "The digital system Artemis for real-time processing of radio transient emissions in the solor corona," Solar Phys., vol. 147, pp. 359-375, 1993

Panagiotis G. Papageorgas was born in Euboia, Greece, in 1961. He received the B.Sc. degree in physics and the Ph.D. degree in applied physics from the University of Athens, Athens, Greece, in 1984 and 1995, respectively. His dissertation research was on the development of sound detection and ranging (SODAR).

From 1985 to 1991, he was a Teaching Assistant in the Laboratories of Electronics and Meteorology, Department of Applied Physics, University of Athens. Since 1994, he has been a Researcher with the Department of Informatics and Telecommunications of the University of Athens in European projects. His main research activity is in the development of electronics and software for instrumentation used in air pollution and biomedical applications, as well as the development of three-dimensional displays, high performance data-acquisition systems, and real-time systems. He is currently an Associate Professor in the Department of Electronics, Technological Educational Institute of Piraeus, Greece.
Dimitris Maroulis was born in Naxos, Greece, in 1949. He received the B.Sc. degree in physics, the M.S. degree in radioelectricity, the M.Sc. in electronic automation, and the Ph.D. degree in informatics, all from University of Athens, Athens, Greece, in 1973, 1977, 1980, and 1990, respectively.

In 1979, he was appointed to Assistant in the Department of Physics and, since 1991 and 1994, he has been Lecturer and Assistant Professor, respectively, in the Department of Informatics, University of Athens, where he currently works with teaching and research activities, including projects with the European community His main areas of activity include signal processing, real-time systems, and development of data acquisition and processing systems for various scientific fields, such as astrophysics and biomedicine.

Harald Winter was born in Oberhausen, Germany, in 1962. He received the B.Sc. degree in physics and the Ph.D. degree in physics, on the use of lasers for gas concentration measurements with the Raman principle, from the Free University of Berlin, Berlin, Germany, in 1992 and 1999, respectively.

From 1988 until 1992, he was a Tutor for geology, medicine, and pharmacy students. From 1993 until 1999, he was a freelance Scientist at the Laser-und Medizin-Technologie GmbH, Berlin.

Stavros A. Karkanis was born in Xanthi, Greece, 1963. He received the B.Sc. degree in mathematics and the Ph.D. degree in informatics from the University of Athens, Athens, Greece, in 1986 and 1995, respectively.

From 1987 to 1995, he was involved in a number of industrial and research projects form various positions in industry and the National Research Center Demokritos. From 1995 to 2002, he was involved, as Senior Consultant, in European-funded research projects with the Department of Informatics, University of Athens. In 2002, he was elected Associate Professor in the Department of Informatics, Technological Educational Institute of Lamia. His main research areas of activity include signal and image processing, pattern recognition, and medical and industrial image processing.

Hansjörg Albrecbt was born in Stuttgart, Germany, in 1942. He received the B.Sc. degree in physics from the University of Stuttgart in 1969 and the Ph.D. degree from the University of Hamburg, Hamburg, Germany, in 1976.

From 1969 until 1971, he was a Development Engineer at AEG-Teleftmken, Berlin, Germany, and from 1971 until 1978, he was a Scientist with the Central Institute for Biomedical Engineering, Erlangen-Nuremberg University, Germany. From 1978 until 1983, he was a Scientist with DFVLR and from 1984 until 1989, he was Head of Product Management Monitoring with Dräger AG, Lübeck, Germany. From 1989 until 1990, he was a Scientific Adviser with Laser-Medizin-Zentrum (LMZ), Berlin, Head of Physicochemical Technologies Department. Since 1990, he has been the Managing Director of the Laser-und Medizin-Technologie GmbH, Berlin.

Nikiforos G. Theofanous was born in loannina, Greece, in 1940. He received the B.Sc. degree in physics, the M.S. degree in radioelectricity, the Ph.D. degree in physics, and the M.Sc. in electronic automation, all from University of Athens, in 1964, 1971, 1973, and 1975, respectively.

In 1969, he was appointed to Assistant and in 1973, he was elected Chief Assistant in the Electronics Laboratory, University of Athens. In 1982, he was elected Lecturer and, in 1984, Assistant Professor in the Department of Physics, University of Athens. In 1990, he was transferred to the Department of Informatics University of Athens, where, in 1991, he was elected Associate Professor and, in 1995, Full Professor of electronics and optoelectronics. He is currently with the Department of Informatics in teaching and research activities, including projects with the European community. His main areas of activity include electrooptics, fiber optics, and optoelectronics with applications in optical communications and in optoelectronic biomedical systems.

Dr. Theofanous is President of the Greek Laser and Electrooptics Scientific Society. 